\title{
Developing gamification e-quizzes based on an android app: the impact of asynchronous form
}

\author{
Marwa F. Areed ${ }^{1} \cdot$ Mohamed A. Amasha $^{2}$ (D) Rania A. Abougalala ${ }^{2}$. \\ Salem Alkhalaf ${ }^{3} \cdot$ Dalia Khairy $^{2}$
}

Received: 16 December 2020 / Accepted: 14 February 2021/ Published online: 24 March 2021

(C) Springer Science+Business Media, LLC, part of Springer Nature 2021

\begin{abstract}
Because of the heath measures taken during the outbreak of Covid-19, the lack of educational methods has become the primary concern among educational professionals who have been using technology as a motivational tool. Gamification is very important because it helps students to represent their study contents and enrich their experiences of higher education when learning in-person is unavailable during the Covid-19 period. This study seeks to present an Android-based gamification app to evaluate the effect of using gamification and e-quizzes on college students' learning. We used the visual blocks language from the MIT App Inventor platform to develop an application, available at (https://play.google.com/store/apps/details?id=appinventor.ai_mekomerofofo. projectGamification). The participants were students from level 2 who used digital lessons for learning MATLAB. The study included gamified learning and non-gamified learning, both integrated into lesson plans, to investigate the differences in learners' performance. Two types of quizzes were used for instruction: gamified e-quizzes and paper-based quizzes. The outcomes plainly showed that using the new gamified e-quiz was more effective than using paper-based quizzes. They are better for assessing the learning performance of the students in question, specifically in terms of formative assessment. It is very important for instructors to apply games as a modern and innovation-oriented tool through which students can be engaged in an attractive, competitive experience.
\end{abstract}

Keywords Gamification · MIT app inventor · Gamified e-quizzes · Formative assessment

\section{Introduction}

It is important to focus on the learning style of each student, because every student has different personality characteristics. One of the essential factors in adapting to learning style with gamification is achieving game balance (Reis et al. 2020). The gamification

Mohamed A. Amasha

mw_amasha@yahoo.com

Extended author information available on the last page of the article 
should not be very simple, because it then becomes boring for students; but it also should not prove too difficult (Mueller et al. 2012). Students may see improvements occurring unequally, creating a risk of separation (Altimira et al. 2014).

Game adaptation in learning includes utilizing the existing knowledge about a student's learning history to provide adaptive learning assistance (e.g., objective formative assessment, other advanced levels more suited to the student's skills). The supportive but challenging character of a game may likely improve motivation and heighten both learning knowledge and skills by allowing for the right difficulty level (for instance, tasks are neither very simple nor very difficult). Thus, these appropriate levels of difficulty ensure that the learner is engaging in activities that are just beyond the student's knowledge but can be performed with supervision (Vargas-Macías et al. 2020; Abougalala et al. 2020). Supervision includes targeted developmental feedback and information to support the learning progress in this gamification task. Studies have shown that learning exercises produce more enjoyable learning results (e.g., Chang et al. 2001; Murphy and Messer 2000)."

Studies show two primary paths by which adapted information is delivered in games: adaptivity takes place both offline and online. For offline adaptivity, context is adapted after collecting enough knowledge about the students before they begin the game (Huang et al. 2020); while with online adaptivity (or dynamic adaptivity), the content is adapted in real-time based on the student's performance. We endorse the second option given that the analysis of learners' competencies will be more accurate when they are executing the task (Shute et al. 2017).

The main purpose of the present research is to examine the impact that a gamified app can have on learning performance specific to students, and how to integrate e-quizzes in a mobile app as a learning tool. Two types of learning method were used: paper-based quizzes with traditional teaching and gamified e-quizzes with a mobile app. The research presents a concept for assessment based on gamification. Gamification has sparked the imagination of people interested in learning, human resource professionals, and others interested in encouraging engagement (Deterding 2012). The current application has proved the effectiveness of gamification on contextual, motivational, and behavioral learning outcomes similar to the study by Sailer and Homner (2020). Gamified equizzes fostered students' social interaction and competition, increasing engagement to enhance learning experiences and motivation and increase attention. In addition, gamified e-quizzes in this paper were found to achieve deep understanding and improve learning, which will lead to better performance in subsequent quizzes. Moreover, gamified equizzes reflect positive effects on current gamification quizzes results. In sum, gamification e-quizzes contribute to the students' feeling of accomplishment and provide a better understanding of the learning progress (Bai et al. 2020).

Based on our theory and literature review, this study seeks to answer the following questions:

What is the impact on students' learning performance of using a mobile app based on gamification as a tool for learning and formative assessment?

1. Is it effective to use a mobile app based on gamification as a formative assessment tool?

2. Does it have a positive effect on students' learning outcomes?

3. What are the differences between using traditional paper quizzes and gamified equizzes in terms of students' learning achievement?" 


\section{Theoretical background}

\subsection{Adaptation of games in learning}

Adaptive gamification can support pleasant and trustworthy conditions intended to make the exercise significant and individually appropriate. With simulations of actual situations and immediate feedback, computer games can provide a practical structure for problems and established knowledge, and hence act as valuable introductions to dynamic, exciting learning. Adding essential background about the students' knowledge to adaptive games can provide better support for relevant and adaptive levels of challenge (Shute et al. 2017).

Some general directions to aggregate data on students through use of gameplay rely on sentiment discovery software and stealth evaluation or infrared cameras. One issue with these options is that many individuals could experience too much pressure if they are already undergoing stress. Therefore, it is difficult to find the task with the right level of pressure. On the other hand, stealth assessment gathers data without obstruction through the game achievements in the game and is free of such bias (Huck et al. 2020).

\subsection{Requirements for an efficient gamification framework}

Gamification used in e-quizzes aims to make the teaching and learning more attractive and motivating in formative assessment (Moreira et al. 2020). Gamification requirements emphasize analyzing the objective needs, practices, and motivations of students (Feger et al. 2019). This section presents two views about the requirements for an efficient gamification framework. The first view consists of five phases of requirements (Heilbrunn et al. 2014):

- Monitoring the gamification phase: to determine the evolution of the gamification application

- Gamification element analytics phase: to explain the development of the game situation and how students communicate with the game parts.

- Gamification design adaptation phase: to test the influence of modifications to the gamification design.

- User groups of interest phase: to focus analyses on subsets of the students.

- Simulation phase: to simulate arbitrary gamification designs with current performance data.

The second view consists the following requirements (Morschheuser et al. 2017):

- Evaluate if gamification is the appropriate method to achieve the objects.

- Know the student demands, motives, and performance, as well as the features of the context. Recognize project aims and describe them precisely.

- Quiz gamification scheme ideas should be as direct as possible.

- Develop an iterative scheme process.

- Develop an intelligent understanding of the game-scheme and learner psychology. 


\subsection{Different options for executing gamification}

Several platforms can be used for gamification; the likely platforms will include affective, cloud-based, and distributed computing.

\subsubsection{Using cloud computing to promote gamification}

Cloud computing allows for a single platform to include the three main services, i.e. infrastructure, software, and platform. This makes it possible for end-users to use the configurable computing devices of their choice. This form of computing can be implemented for gamification by searching every possible configurable resource and using these sources to create and design games with training outcomes. That resolves the difficulty of the cost for educational organizations of improving existing computing facilities (Rahman et al. 2016).

\subsubsection{Applying affective computing through gamification}

Affective computing is considered one of the models likely to also help the gamification concept. The principal object of affective computing is for a computer interface to discover and reply automatically to the user's affect. Emotions are a key characteristic in the creation of games, and recognizing user affects in motion can assist game planners in developing more attractive and suspenseful games. There are many applications of affective computing in the field of artificial intelligence and similar scientific areas. These can be examined for their potential in promoting training within gamification. An interface can be created in this way that can assist learners in improving their educational experience (D'Mello and Calvo 2013)."

\subsubsection{Promoting gamification through distributed computing}

Networking involves transferring data to other modes of communication within computing tools. This is a vast field with wide-ranging applications. In terms of distributed computing, several devices are implemented to process specific requests, which can include multiplayer games, data, or other similar elements. Gamification can be implemented to develop multi-user games in a distributed arena. Therefore, an educational environment based on gamification can support studying between many endusers across various personal workstations (Hakak et al. 2019).

\section{Method}

\subsection{Application design and implementation}

The app was developed with"MIT Software Inventor 2, which consists of a Components Designer and a Blocks Editor. Figure 1 shows the workflow of the application while Fig. 2 shows the segment where the user designs the application's user interface, while the Blocks Editor as seen in Fig. 3 is where the user describes how each user interface part acts using the programming language of Scratch. ". 


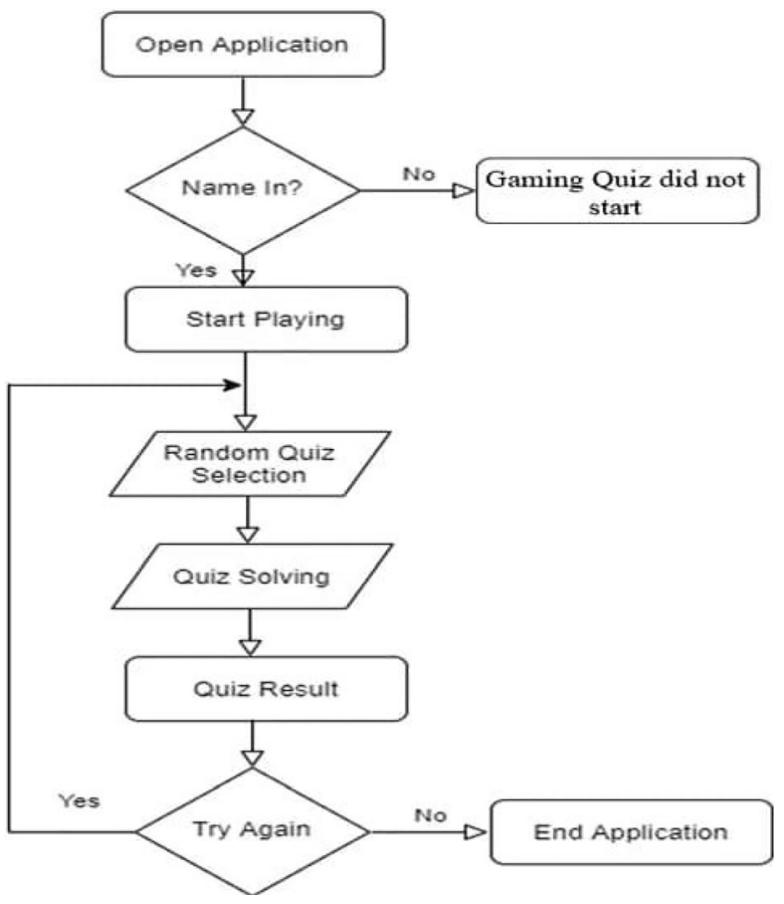

Fig. 1 workflow of the application

\subsubsection{Student login}

The application requires students to login by entering their names the first time only, and every subsequent time they login, their names will appear as shown in Fig. 4; the code for saving students' names and starting the quiz is shown in Figs. 5 and 6.

\subsubsection{Random quiz selection}

This function randomly selects the quiz by spinning the wheel, after which the quiz starts. The quiz random selection screen and its code are shown in Figs. 7 and 8.

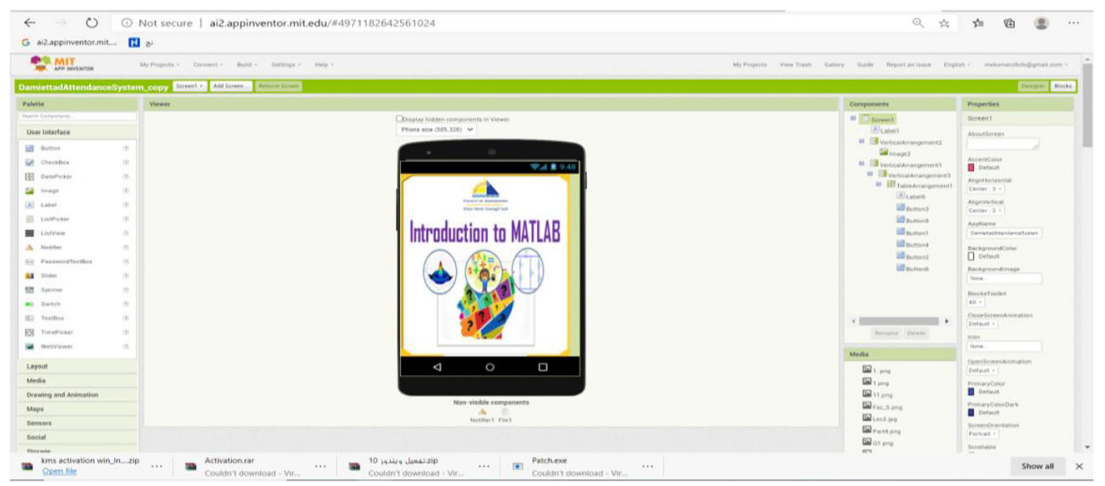

Fig. 2 The Blocks Editor section of MIT App Inventor 2 


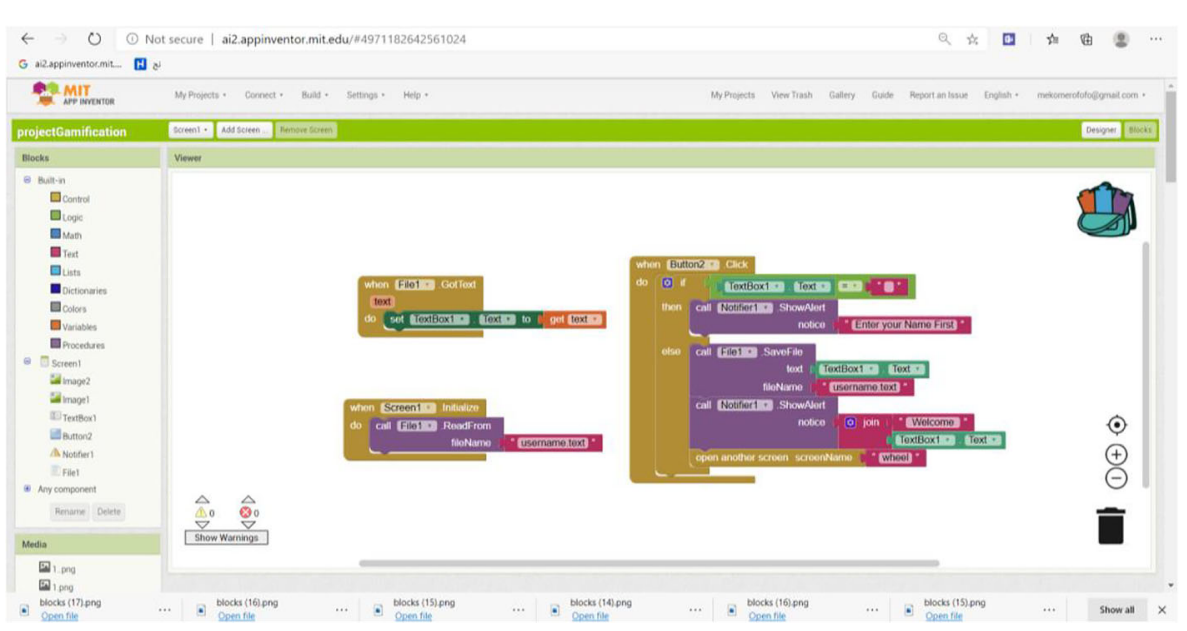

Fig. 3 The Blocks Editor section for block code of MIT App Inventor 2

\subsubsection{Quiz solving}

This part displays the screen for the student to answer the question followed by the nest after answering. The question is randomly chosen, and the available answers are displayed, as shown in Fig. 9 and in the corresponding code shown in Fig. 10.

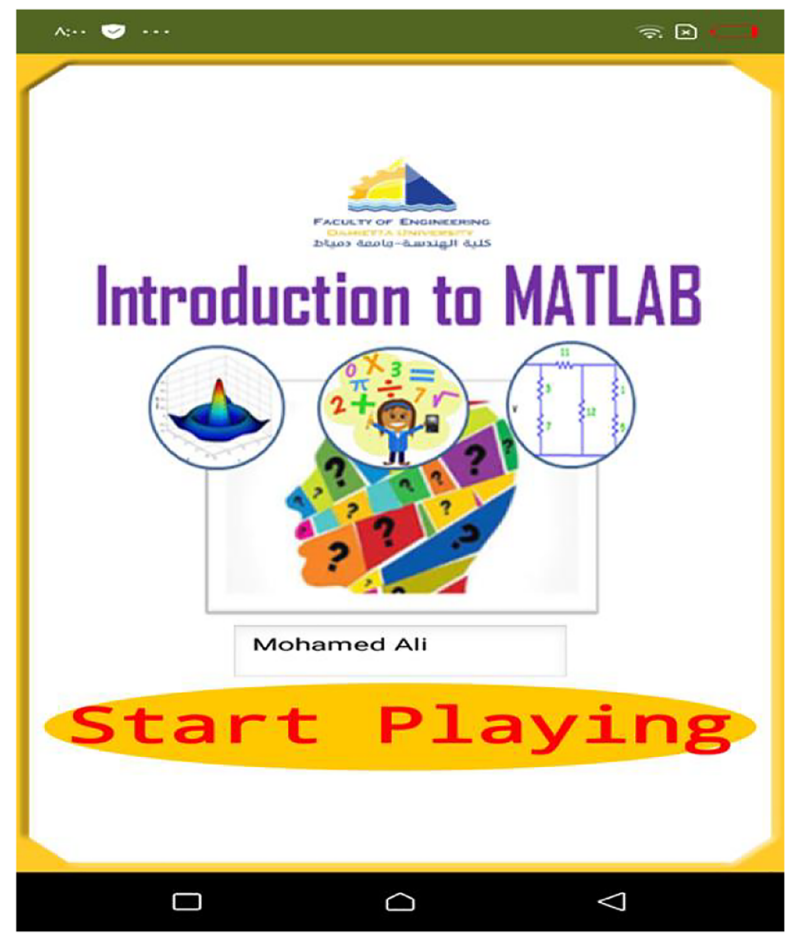

Fig. 4 User interface 


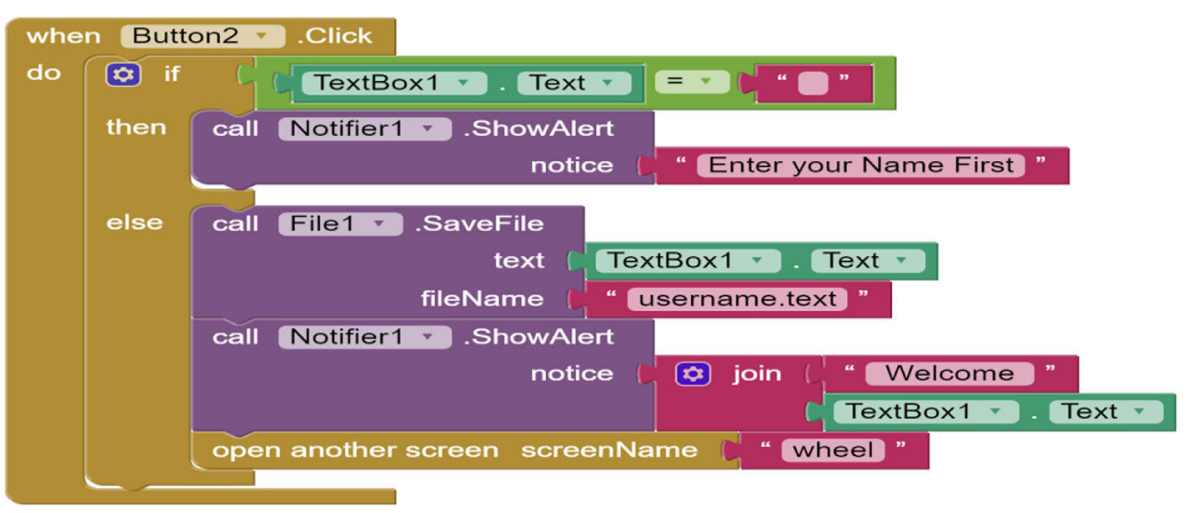

Fig. 5 Logic blocks login code

The code of this part is more complicated and contains all the questions for the chapter and their answers.

Figures $10(a, b, c, d)$ illustrates what happen when the learner clicks on any question choices; the button will be green if the answer is true and red if the answer is false. Figure 10(e) shows the multiple-choice questions (MCQ) of four chapters available in the application and can be extended to include the remaining three chapters of the course. Figures $10(\mathrm{f}, \mathrm{g}, \mathrm{h}, \mathrm{i})$ clarify the basic four programming blocks required for including the chapter questions in the application, whether MCQ or true \& false questions. Figure 10(j) defines the main screen chapters already available with a revision and its possible extension to include three other chapters. Finally, Fig. 10(k) defines the procedure used to show the next question after answering the previous question.

\subsubsection{Answer result and offer of another question}

Finally, the result of the answer is shown as in Fig. 11 and the application gives the user a chance to answer another question or end the application. The code for this task is shown in Fig. 12.

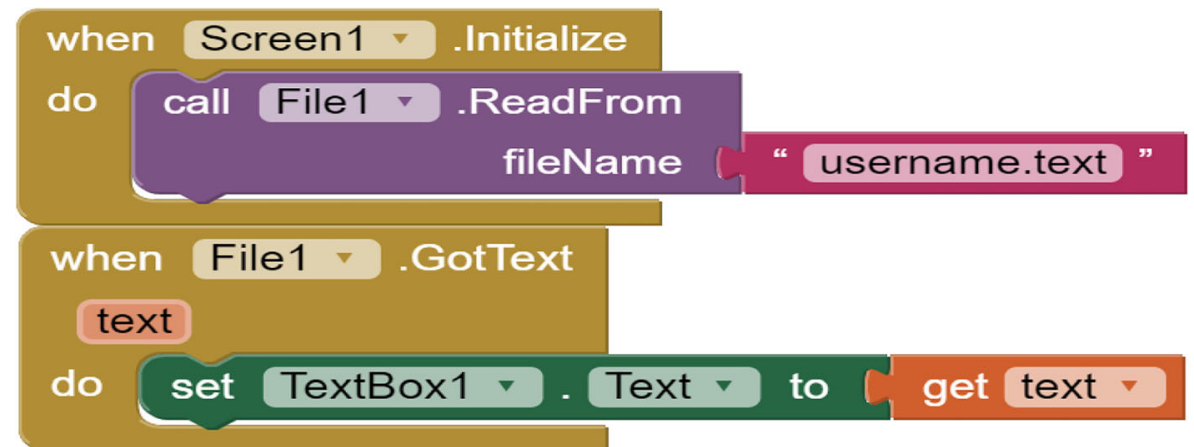

Fig. 6 Logic blocks 


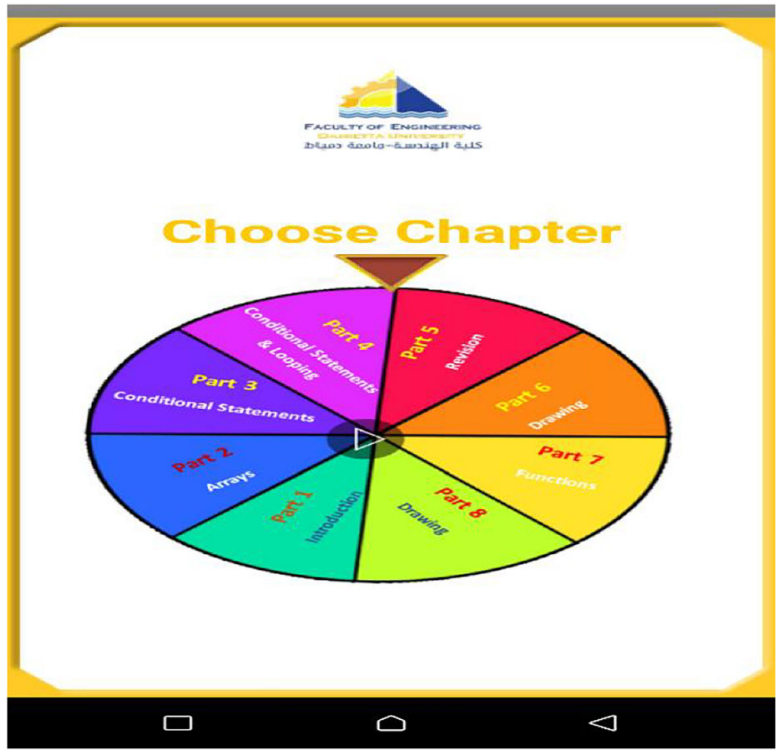

Fig. 7 The random quiz selection screen
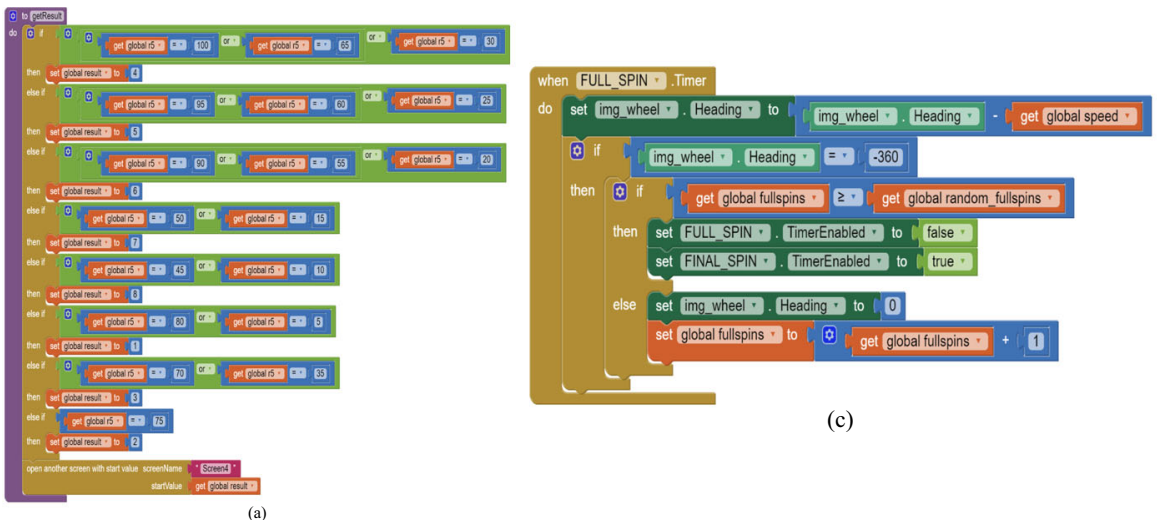

(c)
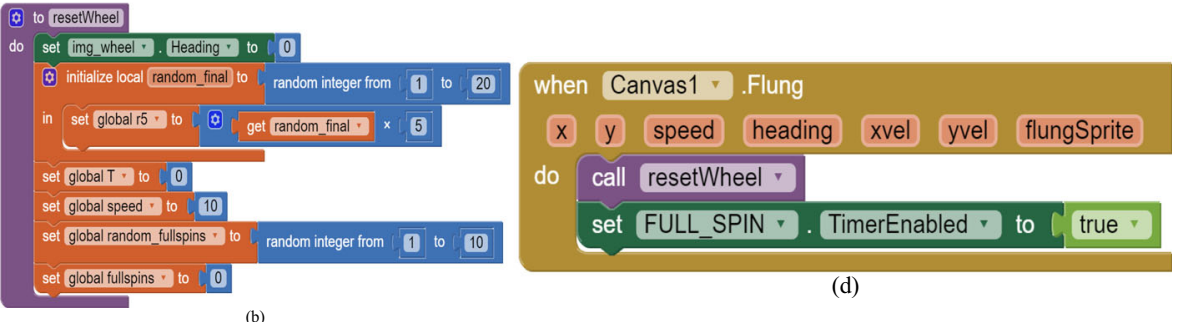

(d)

Fig. 8 Logic blocks for quiz game-play (a,b,c,d) 

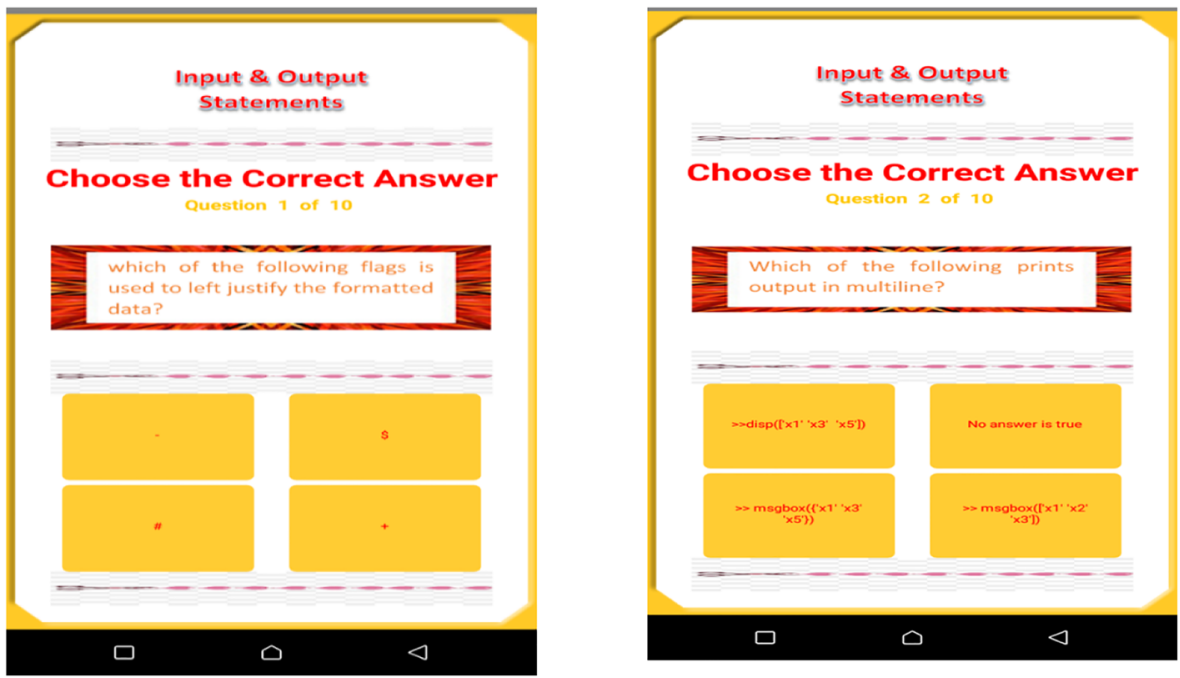

Fig. 9 Question and its answers displayed randomly

\subsection{Participants}

The study randomly selected participants from a pool of 180 students from the Faculty of Engineering at Damietta University. The 60 students selected were all enrolled in their second level and were segmented temporally into an experimental phase and a control phase. Each set had thirty students in it. The experimental group was involved in learning with gamified quizzes, while the other worked with paper quizzes. The experiment was applied to only two units of the MATLAB course over a month within the 2019-2020 academic year. An achievement test was used to quantify the mean scores in math as per the results of both quiz sets (Figs. 13, 14 and 15)."

\subsection{Settings}

We ensured that all students received both types of instruction: gamified and nongamified. One teacher taught the same content to both groups. They had a 45-min meeting once a week. Concerning the course design, both groups participated in the same activities in the class. They began with conventional instruction during the initial meeting, and this was followed with a question and answer session, a paper quiz, and finally the feedback stage. During the next week, one teacher taught the same content to the students (Table 1).

\subsection{Gamified platforms}

The MIT App Inventor language was selected as a platform. It is drag-and-drop visual programming tool for designing and building fully functional mobile apps for Android. The App Inventor supports a new era of personal mobile computing in which users are empowered to develop, create, and deploy individually significant mobile technology solutions for their everyday lives in endlessly unique situations. We used the Blocks 


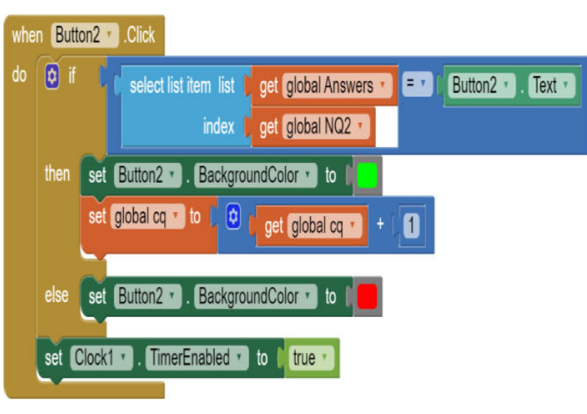

(a)

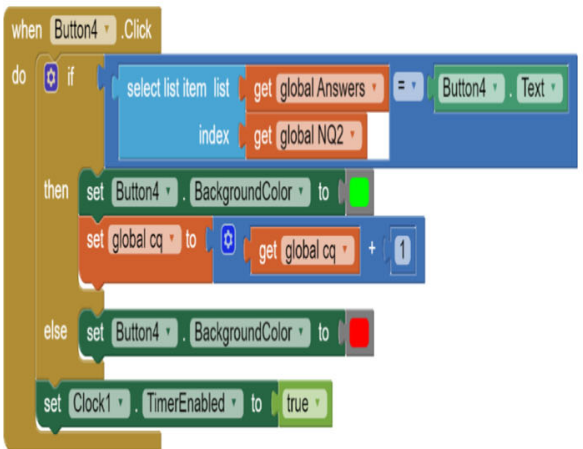

(c)
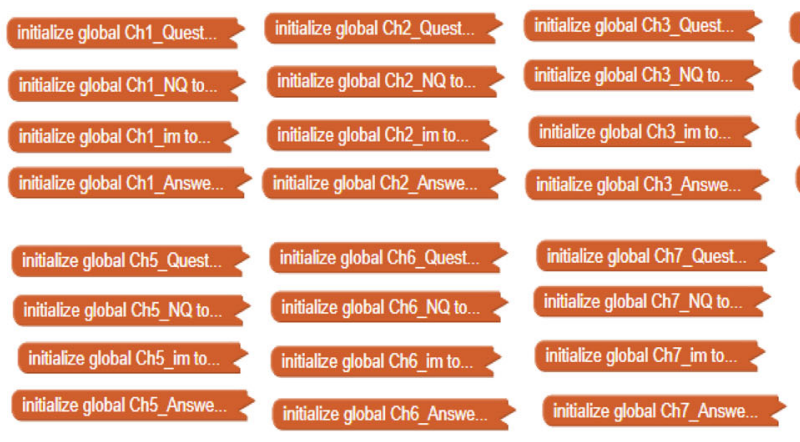

(e)

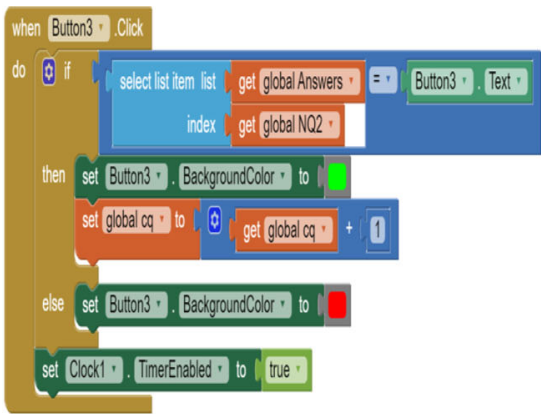

(b)

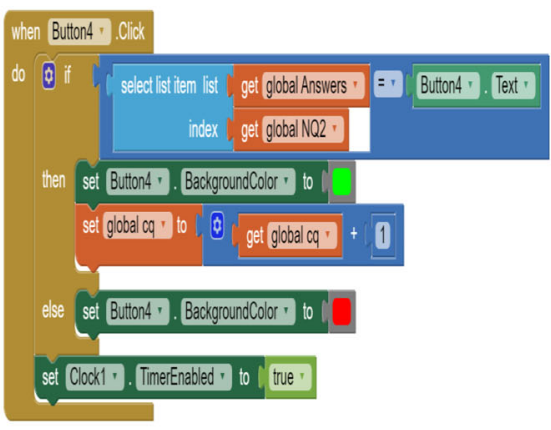

(d) 


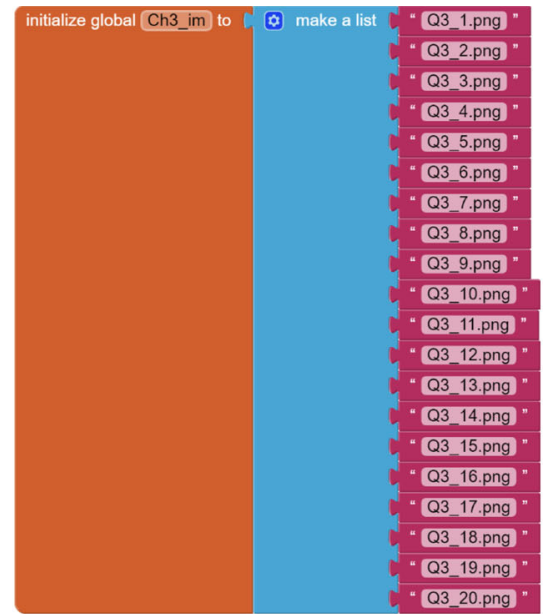

(f)

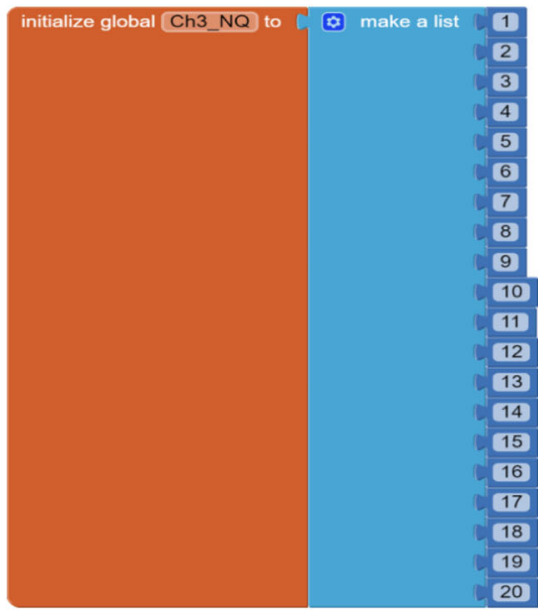

(g)

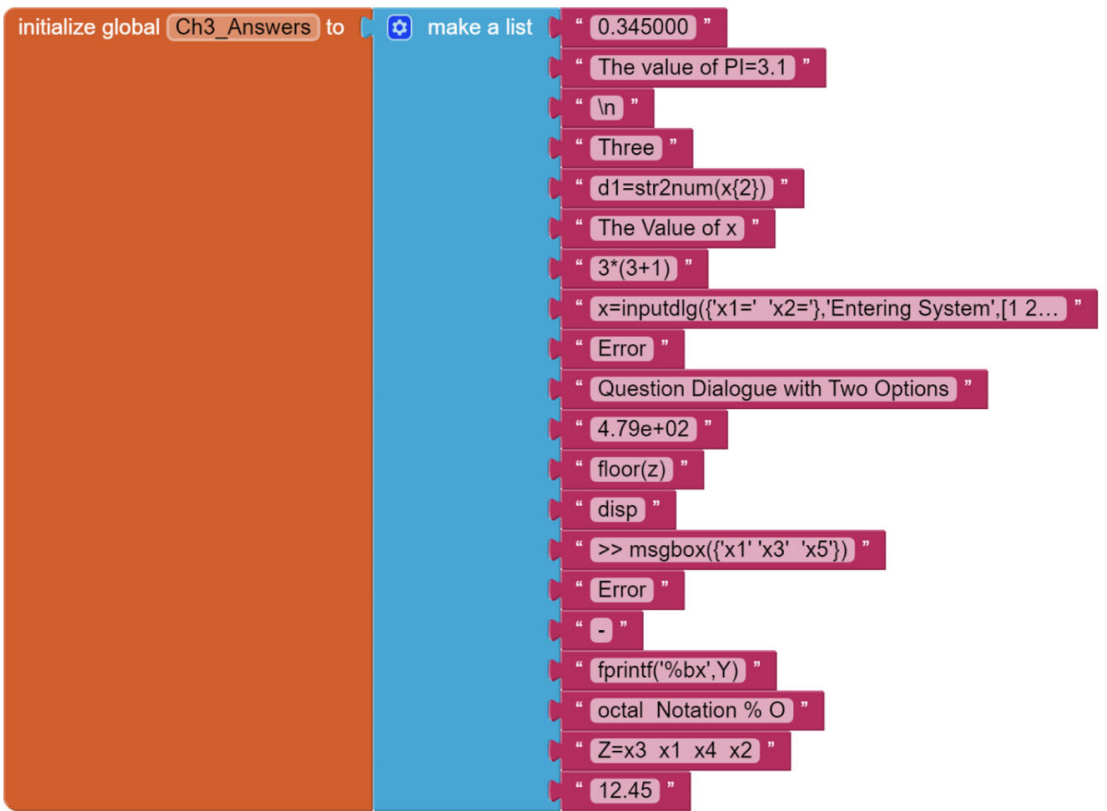

(h)

Fig. 10 (continued)

\subsection{Data analysis}

It took ten weeks to accomplish the research activities. This included familiarizing ourselves with the basic contents of MATLAB, the explanations of the topics, and the assessment activities. The research activities, including preparation, intervention, and assessment activities, were completed during these ten weeks. In week one, participants 


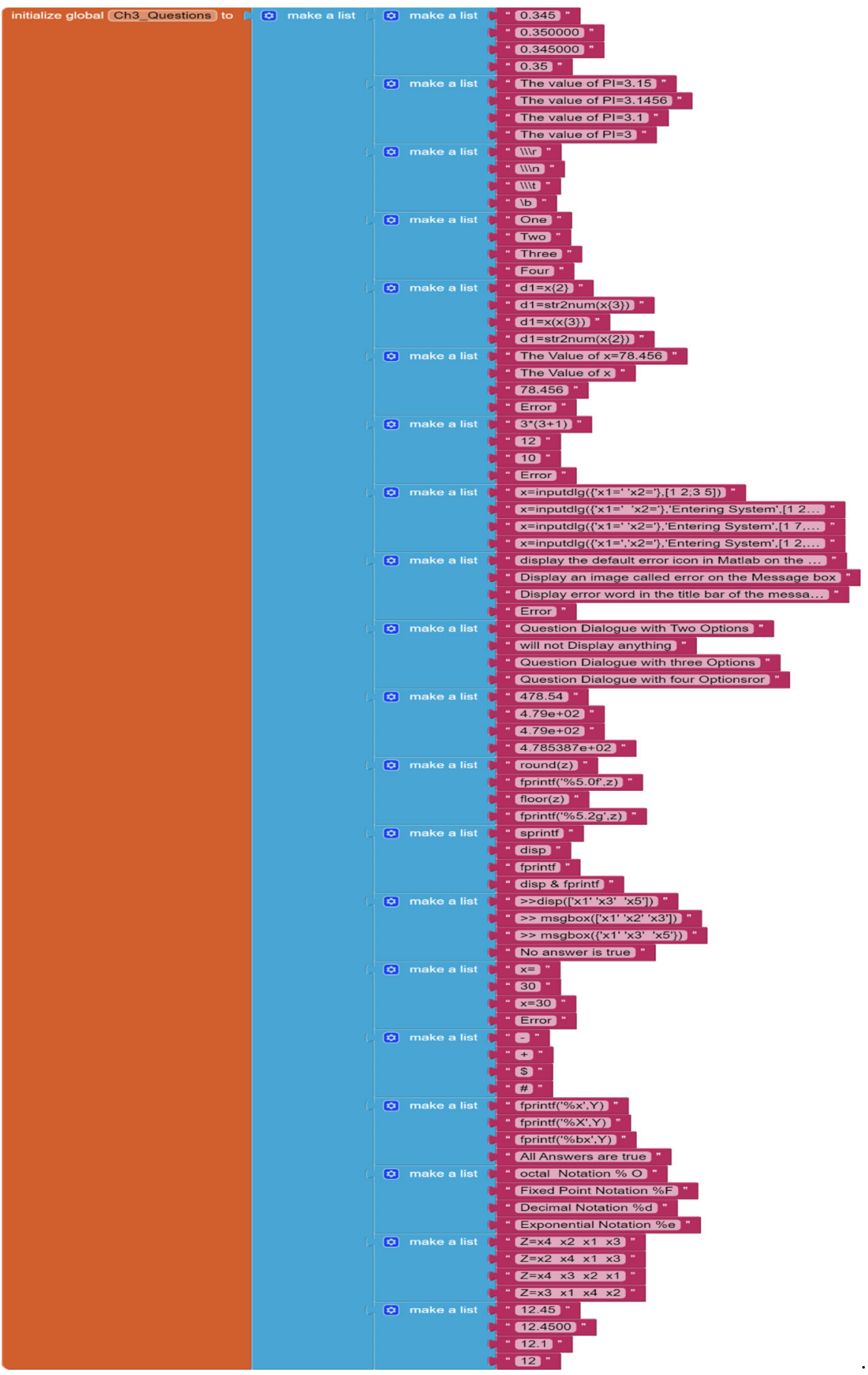

Fig. 10 (continued) 


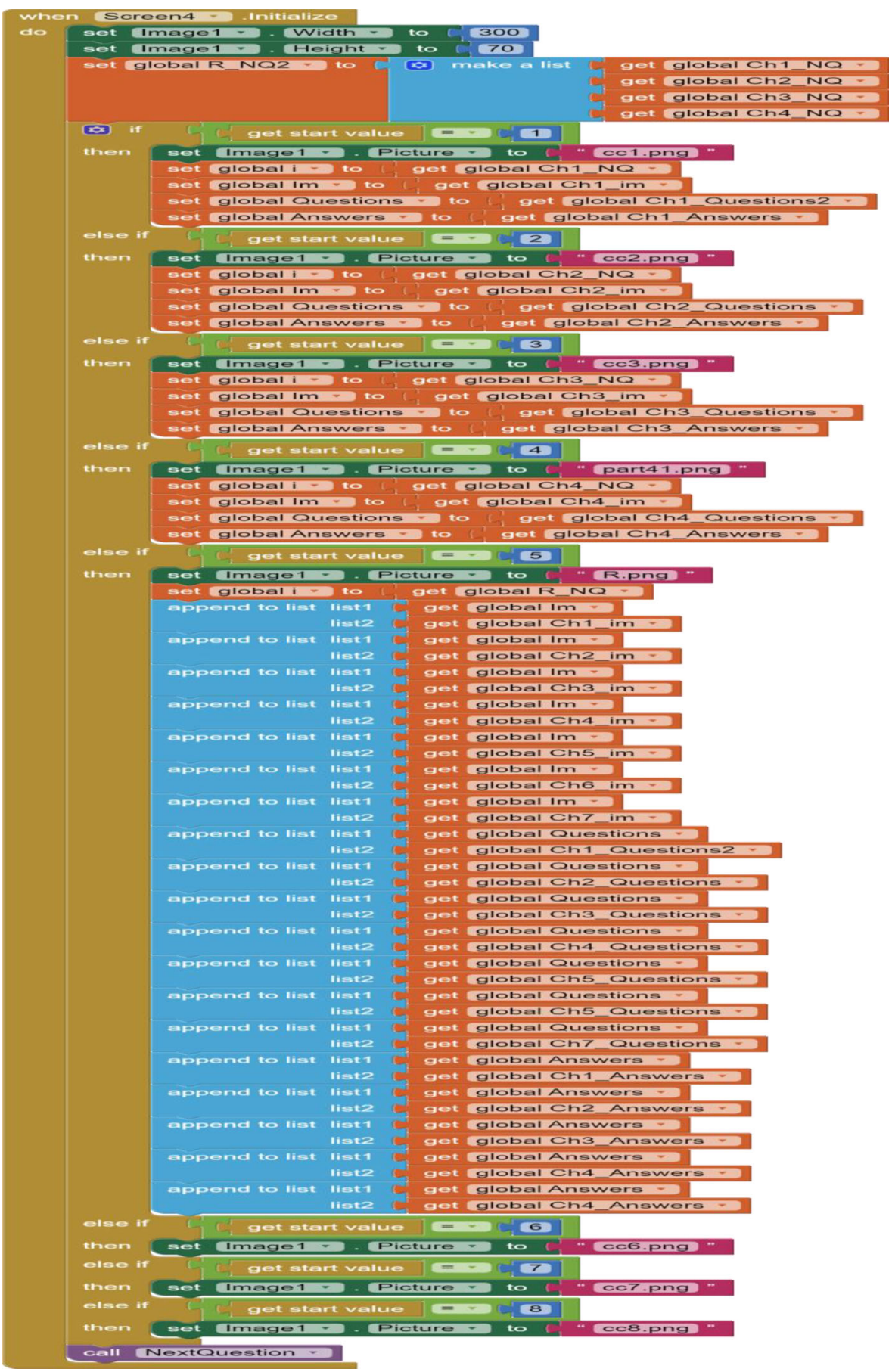

Fig. 10 (continued) 


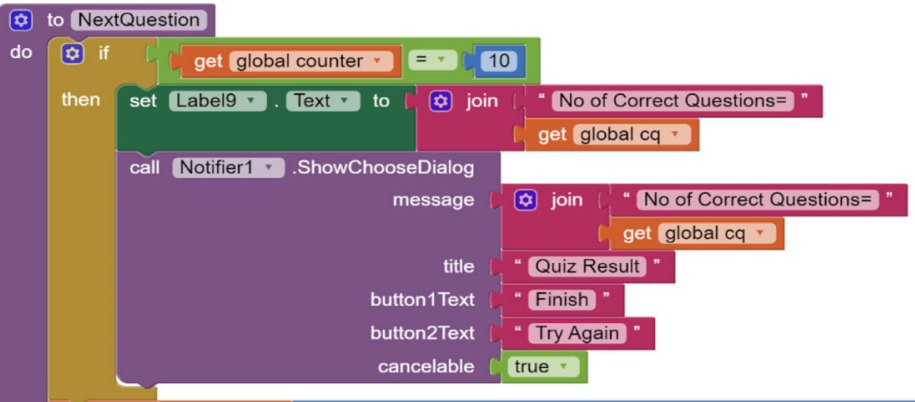

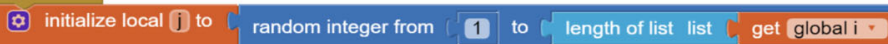

in set global NQ2 + to select list item list get global $\mathrm{i}$

$$
\text { index get } \mathrm{j}=
$$

set global counter * to global counter * +1

set Label8 v. Text $v$ to (\$) join "Question " " of 10 "

set Picturelmage $\cdots$. Picture $\cdots$ to $C$ select list item list 4 get global $\mathrm{Im}$ *

set global values $*$ to select list item list get global Questions

index get global NQ2 *

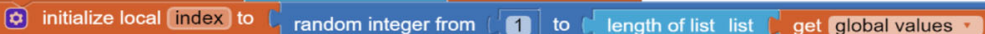

in set Button $\cdots$. Text $r$ to $C$ select list item list get global values $*$

index get index ?

remove list item list get global values *

index get index *

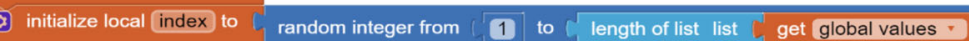

in set Button2 $v$. Text $*$ to $\bar{C}$ select list item list get global values $v$

index get index r

remove list item list get global values .

index get index

initialize local index to random integer from $\sqrt{1}$ to $\sqrt{\text { length of list list get global values }}$.

in set Button $3^{*}$. Text $\cdots$ to $C$ select list item list get global values $*$

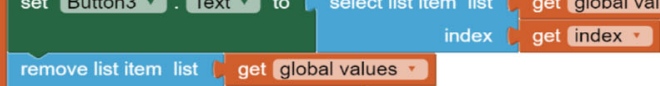

index get index *

initialize local index to random integer from $\sqrt{1}$ to length of list list get global values *

in set Button4 $v$. Text $v$ to $C$ select list item list get global values $v$

index get index
in

remove list item list get global values *

index get index *

remove list item list get global $\mathrm{i}$ -

index get $\mathrm{j}$

set Button1 - BackgroundColor ${ }^{\top}$ to

set Button2 $v$. BackgroundColor $v$ to

set Button3 $r$. BackgroundColor $r$ to

set Button4 * BackgroundColor $*$ to

(k)

Fig. 10 (continued) 


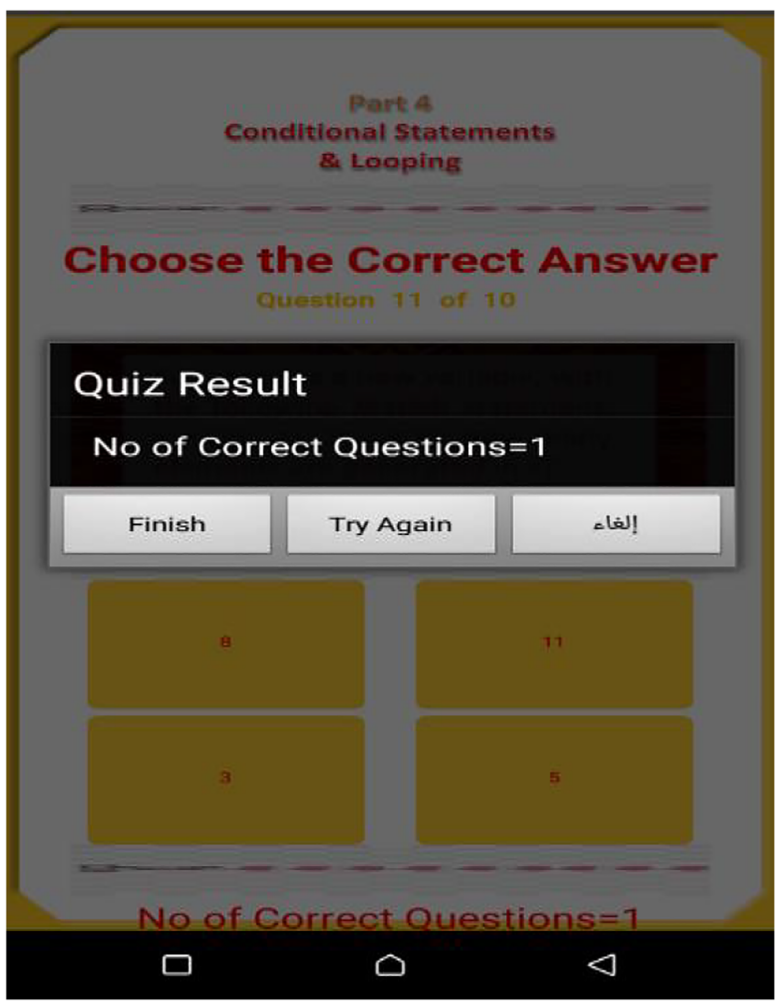

Fig. 11 Answer result screen

were trained and introduced to both the gamified platform and the concept of gamification. Teaching, classroom activities, and exercises were performed starting from week two to week seven. Subsequent to each lecture or at the end of each class

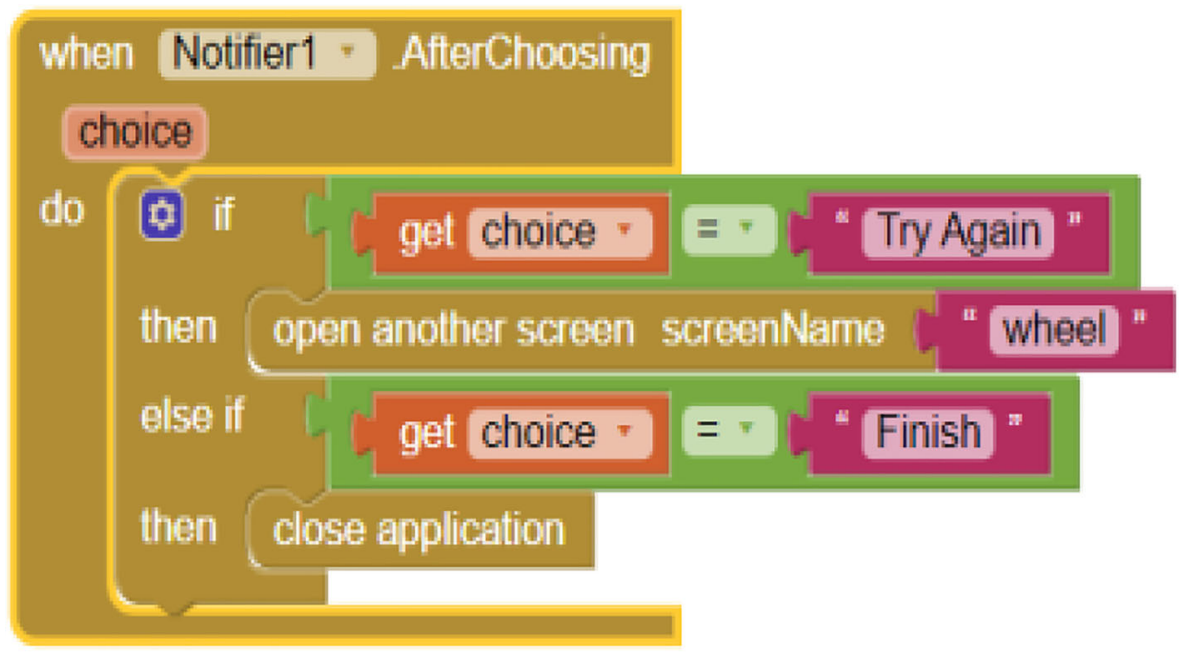

Fig. 12 The code for this task 


\section{PR-POST TEST FOR PAPER-BASED QUIZZES}

$\longrightarrow$ Series1 $\rightarrow$ Series2

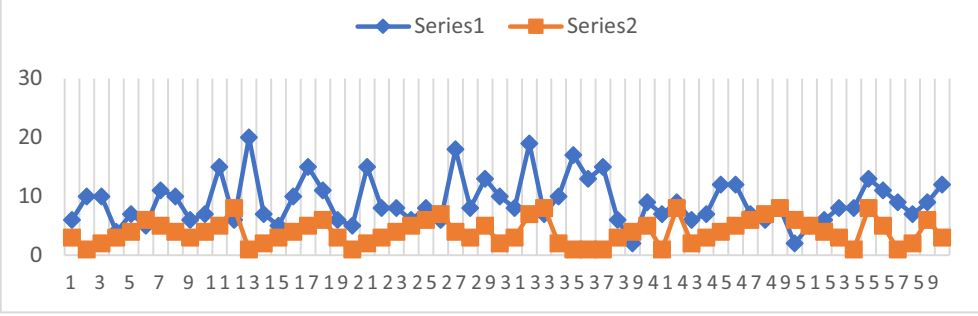

Fig. 13 Pre- and post-test paper-based quizzes

hour, the participants were given two paper-based quizzes. They were also given two gamified e-quizzes on the same content during the following week. Both types of quizzes were made up of 20 questions. Each correct answer gives a score of 1 point, meaning that each quiz had a total score of 20 points. The aim of this mobile app was to evaluate the learners' understanding and assess what has been taught. We used questionnaires and interviews to evaluate the intervention activities during Week Ten."

\section{Results and discussion}

\subsection{Paper-based quizzes}

The conventional instruction method and paper-based quizzes were used with the students' group. A pre-test was conducted to assess the knowledge and skills of the group's students before teaching began. The course took ten weeks, to be taught twice a week. As illustrated in Table 2, the comparison between the results of pre-test and posttest on the students' group showed differences in academic performance for each paperbased quiz as the mean score for the post-test $(\mathrm{M}=9.10, \mathrm{SD}=3.94)$ was considerably higher than that $(\mathrm{M}=3.95, \mathrm{SD}=2.10)$ of the pre-test group. The mean scores of the students' group were significantly different in paper-based assessments $(p<.05)$, and there was a significant difference between their mean scores in the assessment. This result shows that paper-based quizzes still play an important and positive role as formative assessment when evaluating students. Furthermore, gamified e-quizzes supports more effortful retrieval compared to paper quizzes. In addition, gamification e-

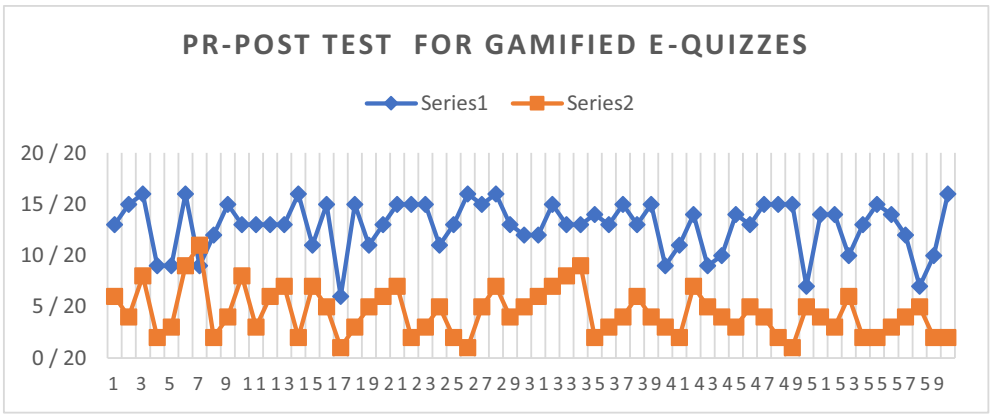

Fig. 14 Pre- and post-test gamified e-quizzes 
COMPARISON BETWEEN GAMIFIED E-QUIZZES VS PAPER-BASED QUIZZES

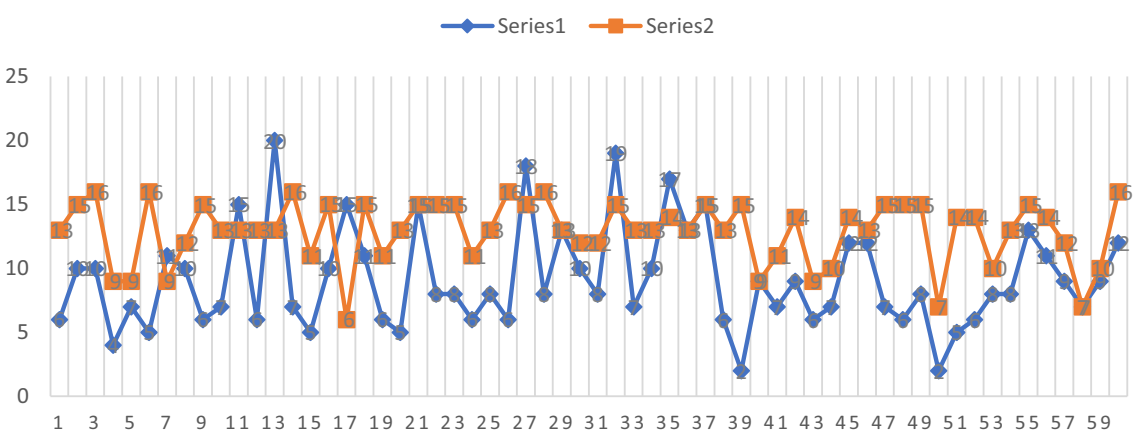

Fig. 15 comparison between gamified e-quizzes vs paper-based quizzes

quizzes foster the neural pathways, expanding the number of neurological connections, which strengthens the availability of the recalled contents of learning better than paper quizzes (Sanchez et al. 2020). Moreover, gamified e-quizzes proved to generate enhanced remembrance of learning after repeated retrieval of that learning compared to limited or no retrieval related to paper quizzes. Additionally, this use of gamification supports the content of the course because of its effectiveness as instructional content; its observed performance points to deeper processing with improved retrieval of knowledge (Putz et al. 2020; Zainuddin et al. 2020). Besides, gamified e-quizzes can be potentially supported by other technology, such as multimedia presentation, interaction means, and attractive design, which compares favorably to paper quizzes.

\subsection{Gamified e-quizzes}

Gamified learning method and gamified e-quizzes were used with the students' group. A pre- and post-test was conducted to assess the knowledge and skills of the group's students before teaching began. The course took ten weeks, to be taught twice a week using online learning by LMS (Microsoft teams). As shown in Table 3, the comparison between pre- and post-test showed differences in academic performance for each gamified e-quiz as the mean score for the post-test $(\mathrm{M}=12.90, \mathrm{SD}=2.49)$ was considerably higher than that $(\mathrm{M}=4.4, \mathrm{SD}=2.28)$ of the pre-test. The results show that there is a difference between the mean scores of pre- and post-test. The mean

Table 1 Design of the course with traditional learning and online learning

\begin{tabular}{lll}
\hline & Traditional Learning & Online Learning \\
\hline Lectures & Face to face learning in the university & Using Zoom or videos on YouTube \\
Quizzes & - Short paper quizzes & - Online quizzes with a large number of questions \\
& - The same for all students & - Different for each student \\
& - Questions and their answers are static & - Questions and their answers are randomly displayed \\
& - The result appears after one week at least. & - The quiz result is displayed immediately. \\
\hline
\end{tabular}


Table 2 The $t$-test values for pr-post test for paper-based quizzes

\begin{tabular}{llllll}
\hline Group & $M$ & $S D$ & $D F$ & $t$ & Sig. \\
\hline Pre-test & 3.95 & 2.10 & 59 & 17.85 & 0.000 \\
Post-test & 9.10 & 3.94 & & & \\
\hline
\end{tabular}

${ }^{*} p<0.05 . * * p<0.01$

scores of pre- and post-test were significantly different in gamified e-quiz assessments $(p<.05)$, and there was significant difference between their mean scores in assessment. This result shows that gamified-based quizzes helps students to take on a more a significant role in evaluating students and in increasing academic performance in formative assessment.

\subsection{Gamified via paper-based quizzes}

The primary purpose of this research is to compare the use of gamified e-quizzes and paper-based quizzes in assessing student academic performance through formative assessment. Paper-based quizzes were taken in the traditional way after teaching concluded, while gamified e-quizzes were taken by using a mobile app after teaching concluded. The results in Table 4 compare the academic performance between gamified e-quizzes and paper-based quizzes, showing that the mean achievement score for the gamified e-quizzes group $(M=12.90, S D=2.49)$ was considerably higher than that $(M=9.10, S D=2.49)$ of the paper-based quizzes group. The results indicated that there is difference between the mean scores of the two groups, gamified and paper. The mean scores of the group were significantly different in gamified e-quiz assessments ( $p$ $<.05$ ), and there was a significant difference between the pre and post-test in mean scores in assessment. This result shows that gamified e-quizzes help students to evaluate academic performance in formative assessment, provide feedback to students, improve their knowledge, and support enjoyable and competition-based learning. These results were in agreement with the recommendations of previous studies that e-evaluation can be relied upon in evaluating students after teaching. Basically, Kapp (2012) implied that gamification is established in fundamental instructional methods and practices while offering a contemporized approach to feedback, rewards, and encouragement. Nonetheless, it should be noted that the current appraisal framework has put a lot of pressure on the contemporary student; despite the fact that the focus on assessment may inspire many, reports have indicated that undergraduate students are increasingly overwhelmed and stressed by academic pressures related to grades and

Table 3 The t-test values for pre- and post-test for gamified e-quizzes

\begin{tabular}{lrllll}
\hline Group & $M$ & $S D$ & $D F$ & $T$ & Sig. \\
\hline Pre-test & 4.4 & 2.28 & 59 & 40.04 & 0.000 \\
Post-test & 12.9 & 2.49 & & & \\
\hline
\end{tabular}

${ }^{*} p<0.05 . * * p<0.01$ 
Table 4 Comparison between gamified e-quizzes and paper-based quizzes

\begin{tabular}{lccccc}
\hline Group & $M$ & $S D$ & $D F$ & $t$ & Sig. \\
\hline Group 1(paper) & 9.10 & 3.94 & 118 & 6.303 & 0.000 \\
Group 2 (gamified) & 12.9 & 2.49 & & & \\
\hline
\end{tabular}

${ }^{*} p<0.05 .{ }^{* *} p<0.01$

testing (Smith and Abrams 2019). While gamification is a strategy to promote learning utilizing game-related elements that offer important criticism and collaboration, unbending and rigid educational programs and appraisals impose barriers on some students, especially the individuals who are distinguished as who have learning disabilities. (Dalton 2017; Everett and Oswald 2018). Moreover, when feedback and motivation are primarily point-driven, the path toward learning might become subservient to finding the "right" answer.

\section{Conclusion}

Gamification is fundamental to helping students continue with the learning process during the current COVID-19 pandemic. As shown in the research, gamification in the form of e-quizzes is aimed at making the teaching and learning process not only motivating but also attractive based on formative assessment. Accordingly, gamification requires an analysis of the objective needs, practices, and motivations of students. In this study, two types of learning methods were used: paper-based quiz learning through traditional teaching and gamified e-quizzes using a mobile app. Consequently, the primary purpose of this research was to compare the use of gamified e-quizzes and paper-based quizzes in examining the participants' academic performance as a formative assessment.

In terms of learning performance, the findings of the pre- and post-tests reveal that the scores in the gamified e-quizzes were higher compared to those of the students who participated in traditional paper-based quizzes, especially in the post-tests. Accordingly, the results also show that the students who participated in the gamified e-quizzes demonstrated more competency than those who participated in paper-based quizzes. This is shown by the higher mean scores of students who took e-quizzes using a mobile app after the completion of the course. Consequently, results from students in the gamified e-quizzes demonstrated increased competency when it came to both learning and the mastery of new skills in and out of the classroom.

Evidently, the results show that students who engaged with the gamified e-quizzes were not only adept at using technology but were also able to think critically. During the experiments, students who participated in the gamified e-quizzes acknowledged that learning by using the gamified app improved their critical thinking based on how the questions made them think outside of the box. Before attending classes, students were encouraged to go through course materials in order to improve their scores. Using the gamified app, students increased their understanding of the course materials, which were demonstrated in the high scores of the gamified e-quizzes. In this case, the 
students who participated in the gamified e-quizzes demonstrated more learning capabilities as compared to the students using traditional paper-based quizzes.

In addition to the students' competency, the gamified e-quizzes approach also improved their autonomy in the learning process. The gamified learning platform provided a flexible learning environment for creating and establishing studentcentered learning, which helped develop autonomous learners. As depicted in the quantitative analysis, the mean scores of students learning through gamified equizzes were higher than those of traditional paper-based quizzes. This reveals that the e-quizzes provided students with the capacity to work through the course at their comfortable pace. Moreover, students were also able to study both in and out of the classroom, which added to the gamified app's flexibility. For instance, students controlled when they were learning by taking the quizzes when it was convenient for them.

Consequently, we can say in summary that the gamified e-quizzes provided more flexibility that made the students feel more competent and in charge of their learning and assessment process. The gamified app provides learners with more opportunities to prepare and learn pre-course materials before going to class, as well as be better prepared for the quiz activities by completing them at their most convenient time. Thus, the results from the research were in agreement with the recommendations of previous studies that e-evaluation can be relied upon in evaluating students after teaching.

\section{References}

Abougalala, R. A., Amasha, A., Areed, M. F., Alkhalaf, S., \& Khairy, D. (2020). Blockchain-enabled smart university: A framework. Journal of Theoretical and Applied Information Technology, 98(17), 35313543.

Altimira, D., Mueller, F. F., Lee, G., Clarke, J., Billinghurst, M. 2014. Towards understanding balancing in exertion games. In Proceedings of the 11th Conference on Advances in Computer Entertainment Technology (ACE '14). Association for Computing Machinery, New York, NY, USA, Article 10, 1-8. https://doi.org/10.1145/2663806.2663838.

Bai, S., Hew, K. F., \& Huang, B. (2020). Does gamification improve student learning outcome? Evidence from a meta-analysis and synthesis of qualitative data in educational contexts. Educational Research Review, 30, 100322.

Chang, K. E., Sung, Y. T., \& Chen, S. F. (2001). Learning through computer-based concept mapping with scaffolding aid. Journal of Computer Assisted Learning, 17(1), 21-33.

Dalton, E. M. (2017). Beyond universal Design for Learning: Guiding principles to reduce barriers to Digital \& Media Literacy Competence. Journal of Media Literacy Education, 9(2), 17-29.

Deterding, S. (2012). Gamification: Designing for motivation. Interactions, 19(4), 14-17.

D'Mello, S., \& Calvo, R. A. (2013). Beyond the basic emotions: what should affective computing compute? In CHI'13 Extended Abstracts on Human Factors in Computing Systems (CHI EA'13) (pp. 2287-2294). New York, NY, USA: Association for Computing Machinery. https://doi.org/10.1145/2468356.2468751.

Everett, S., \& Oswald, G. (2018). Engaging and training students in the development of inclusive learning materials for their peers. Teaching in Higher Education, 23(7), 802-817.

Feger SS, Dallmeier-Tiessen S, Woźniak PW, \& Schmidt A. (2019). Gamification in science: a study of requirements in the context of reproducible research. In Proceedings of the 2019 CHI Conference on Human Factors in Computing Systems (CHI'19) (pp. 1-14). New York, NY, USA: Association for Computing Machinery, Paper 460. https://doi.org/10.1145/3290605.3300690.

Hakak, S., Noor, N. F. M., Ayub, M. N., Affal, H., Hussin, N., \& Imran, M. (2019). Cloud-assisted gamification for education and learning-recent advances and challenges. Computers \& Electrical Engineering, 74, 22-34. 
Heilbrunn, B., Herzig, P. \& Schill, A., (2014). Towards gamification analytics - requirements for monitoring and adapting gamification designs. In: Plödereder, E., Grunske, L., Schneider, E. \& Ull, D. (Hrsg.), Informatik 2014. Bonn:Gesellschaft für Informatik e.V. (S. 333-344).

Huang, F., Cao, M., \& Wang, L. (2020). Supplementary material from Learning enables adaptation in cooperation for multi-player stochastic games. The Royal Society Collection. https://doi.org/10.6084/ m9.figshare.c.5202170.v2.

Huck, J. T., Day, E. A., Lin, L., Jorgensen, A. G., Westlin, J., \& Hardy III, J. H. (2020). The role of epistemic curiosity in game-based learning: Distinguishing skill acquisition from adaptation. Simulation \& Gaming, 51(2), 141-166.

Kapp, K. M. (2012). The gamification of learning and instruction: game-based methods and strategies for training and education. John Wiley \& Sons. Published by Pfeiffer.

Moreira, F., Ferreira, M. J., Escudero, D. F., Pereira, C. S., \& Durão, N. (2020). Teaching and learning Modelling and Specification based on gamification (pp. 1-6). Seville, Spain: 2020 15th Iberian Conference on Information Systems and Technologies (CISTI). https://oi.org/10.23919/CISTI49556. 2020.9140829.

Morschheuser, B., Hamari, J., Werder, K., \& Abe, J. (2017). How to gamify? A method for designing gamification. In Proceedings of the 50th Hawaii International Conference on System Sciences (HICSS) 2017. Manoa: University of Hawai'i. https://doi.org/10.24251/HICSS.2017.155.

Mueller, F., Vetere, F., Gibbs, M., Edge, D., Agamanolis, S., Sheridan, J., \& Heer, J. (2012). Balancing exertion experiences. In Proceedings of the SIGCHI Conference on Human Factors in Computing Systems (CHI '12) (pp. 1853-1862). New York, NY, USA: Association for Computing Machinery. https://doi.org/10.1145/2207676.2208322.

Murphy, N., \& Messer, D. (2000). Differential benefits from scaffolding and children working alone. Educational Psychology, 20(1), 17-31.

Putz, L. M., Hofbauer, F., \& Treiblmaier, H. (2020). Can gamification help to improve education? Findings from a longitudinal study. Computers in Human Behavior, 110, 106392.

Rahman, M. N. A., Saidu, A. N., Kadir, M. F. A., Shamsudin, S. N., \& Saany, S. I. A. (2016). A gamification model for resource sharing in Malaysian schools using cloud computing platform. In International Conference on Intelligent Systems Design and Applications (pp. 406-416). Cham: Springer.

Reis, S., Reis, L. P., \& Lau, N. (2020). Game adaptation by using reinforcement learning over meta games. Group Decision and Negotiation. https://doi.org/10.1007/s10726-020-09652-8.

Sailer, M., \& Homner, L. (2020). The gamification of learning: A meta-analysis. Journal of Educational Psychology Review, 32, 77-112.

Sanchez, D. R., Langer, M., \& Kaur, R. (2020). Gamification in the classroom: Examining the impact of gamified quizzes on student learning. Computers \& Education, 144, 103666.

Shute, V., Ke, F., \& Wang, L. (2017). Assessment and adaptation in games. In Instructional techniques to facilitate learning and motivation of serious games (pp. 59-78). Cham: Springer.

Smith, K., \& Abrams, S. S. (2019). Gamification and accessibility. International Journal of Information and Learning Technology, 36(2), 104-123. https://doi.org/10.1108/IJILT-06-2018-0061.

Vargas-Macías, Z. L., Rodríguez-Hernández, A. A., \& Sánchez-Sáenz, C. L. (2020). Digital games (Gamification) in learning and training: An approach to adaptation and integration in the classroom. GIST-Education and Learning Research Journal, 20, 171-188.

Zainuddin, Z., Shujahat, M., Haruna, H., \& Chu, S. K. W. (2020). The role of gamified e-quizzes on student learning and engagement: An interactive gamification solution for a formative assessment system. Computers \& Education, 145, 103729.

Publisher's Note Springer Nature remains neutral with regard to jurisdictional claims in published maps and institutional affiliations. 


\section{Affiliations}

Marwa F. Areed ${ }^{1} \cdot$ Mohamed A. Amasha $^{2} \cdot$ Rania A. Abougalala ${ }^{2} \cdot$ Salem $^{-}$ Alkhalaf $^{3}$ - Dalia Khairy ${ }^{2}$

Marwa F. Areed

Marwa_Areed@du.edu.eg

Rania A. Abougalala

Ronyabogalala@hotmail.com

Salem Alkhalaf

s.alkhalaf@qu.edu.sa

Dalia Khairy

shamaamora2014@gmail.com

1 Faculty of Engineering, Department of Electrical Engineering, Damietta University, Damietta, Egypt

2 Faculty of Specific Education, Department of Computer Teacher Preparation, Damietta University, Damietta, Egypt

3 Faculty of Science and Arts, Computer Science Department, Qassim University, Alrass, Saudi Arabia 